\title{
Necrotizing Fasciitis Secondary to Vibrio vulnificus
}

\author{
Hossein Akhondia, c, Adriana Garza Lopez ${ }^{\mathrm{b}}$
}

\begin{abstract}
Necrotizing fasciitis is rare, quickly progressive, hard to diagnose, difficult to treat, with poor outcomes and therefore poses serious medical and occasionally legal concerns. We present a unique patient with no past medical history who had an infection with Vibrio vulnificus after a marine accident. Only amputation saved his life.
\end{abstract}

Keywords: Necrotizing fasciitis; Vibrio vulnificus; Salt-water injury; Polymicrobial infection; Soft tissue infection and gangrene

\section{Introduction}

Necrotizing fasciitis was first introduced as "streptococcal gangrene" in 1924, later called "Melaney's gangrene". In 1952 the term "necrotizing fasciitis" was given to the signs and symptoms presented by these patients. In 1977 Giuliano and colleagues recognized that more than one pathogen could be involved in this entity.

Necrotizing fasciitis is rare, quickly progressive, and spreads throughout the skin and subcutaneous tissues. It may be polymicrobial (type I), which is $98 \%$ of cases or monomicrobial infection (type II). The most common agents are group A Streptococcus, methicillin-resistant Staphylococcus aurous, Clostridium perfringens, Bacteroides fragilis and Aeromonas hydrophilia. More than $70 \%$ of the patients have a past medical history or an immune-compromised state and mortality rate can be up to $75 \%$. Treatment must include early intravenous antibiotics and aggressive surgical debridement. If left untreated or if treatment is delayed, it will expand and cause systemic toxicity, sepsis and shock.

Manuscript accepted for publication November 17, 2014

${ }^{a}$ Georgetown University School of Medicine, 1010 Massachusetts Ave, NW, Unit 904, Washington, DC 20001, USA

${ }^{\mathrm{b}}$ Monterrey, Mexico

${ }^{c}$ Corresponding Author: Hossein Akhondi, Georgetown University School of Medicine, 1010 Massachusetts Ave, NW, Unit 904, Washington, DC 20001, USA.Email: h68akhond@hotmail.com

doi: http://dx.doi.org/10.14740/jmc2026e
Although rare, necrotizing fasciitis may be caused by $\mathrm{Vi}$ brio vulnificus, a gram-negative bacterium of the family $\mathrm{Vi}$ brionaceae, which is an inhabitant of salt water. This is more prevalent in patients with chronic liver disease as well as other immune-compromising states, and is mainly induced by direct injury from the fish backbone while purchasing, cleaning or handling live fish [1].

\section{Case Report}

Our patient was a 50-year-old white male with no past medical history, taken to emergency room 4 days after feeling a foot bite while fishing without shoe in a "lake" in state of Georgia. At the hospital the lesion of left foot was observed to be red and inflamed and thought to be cellulitis. There was moderate leukocytosis of 15,000 but no other lab abnormality and no gas or fracture in the X-rays. Treatment with third generation cephalosporin started intravenously. Lesion worsened, and pain ensued and areas of blackening were noticed around the area 1 day later (Fig. 1). Blood and wound cultures grow $\mathrm{Vi}$ brio vulnificus, which was interesting because it is a salt-water pathogen and it was not suspected in this lake-fisherman. Upon more questioning he reported that the "lake" was in fact connected with sea water. Fluroquinolone was added, but patient got worse with tachycardia and fever. He was taken to operating room, and a mid-ankle amputation was done with resolution of symptoms. He did not develop further complications nor did he require a secondary amputation.

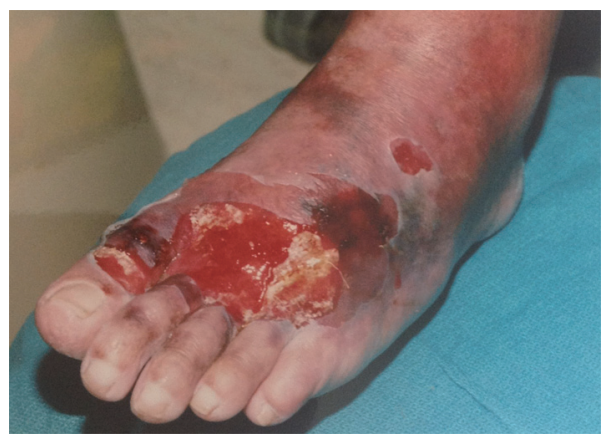

Figure 1. Extremely inflamed and necrotic foot. 


\section{Discussion}

With the ongoing increase of immune-compromised patients, necrotizing fasciitis has become more common. They usually have an underlying problem. In one study of 102 cases, $43.1 \%$ had diabetes mellitus, $23.5 \%$ trauma, $23.5 \%$ intravenous drug abuse, $3.9 \%$ surgery and $1.9 \%$ malignancy. Of these patients, the area mostly involved was genital and perianal area $(49 \%)$, upper and lower limb (43.1\%) and lower abdomen (33.3\%). Rapid progression is characteristic of necrotizing fasciitis so rapid treatment is imperative. In the same study, the muscles were involved in 26 patients, and extensive surgical debridement was required in 90 patients [2].

Infection by Vibrio vulnificus is related to poor hygiene mainly presented in the middle-age adult, and is rapidly progressive. No sex prevalence has been recognized. Septicemia by Vibrio vulnificus causes $95 \%$ of seafood related deaths [3]. Necrotizing fasciitis due to Vibrio is a rare culminating stage of previous cellulitis caused by eating raw food or having an open wound. Currently in the US, there are about 50 cases per year documented and the majority is found in the Golf coast region [4]. As it is evident by this case, the history can be confusing, and good epidemiology knowledge and familiarity with this infection especially in Gulf coast region are helpful.

A previous study of 132 cases involving Vibrio vulnificus biotype 3 with severe soft tissue infection after fishbone injury was done in Israel between 1996 and 1997. Out of 62 patients there was a slight male predominance of $58 \%$ and a median age of 56. No deaths were reported, and $66 \%$ required surgical debridement. One patient had total limb amputation. A study of 134 patients was done from 1998 to 2005 . The source of infection was found in wounds of 61 patients, blood of 24 patients and in both in seven patients (two patients were not recorded). Of the wounded ones, $52.56 \%$ were injured while purchasing or preparing the fish, $9.10 \%$ while mobile selling and $14.15 \%$ while standing near fish ponds. Out of all the patients, 124 required hospitalization with a mean duration of stay of 10.9 days. One hundred twelve patients required a single drug (47 with ampicillin-clavulanate, 27 doxycycline, six ceftriaxone). The rest required combined treatment [1].

Collins did a study specifically focusing on Vibrio infection and blue mussels in 2011 [5]. Blue mussels were investigated for occurrence of Vibrio cholera, Vibrio parahaemolyticus and Vibrio vulnificus in the sound between Sweden and Denmark. The $53 \%$ of mussel samples were positive for Vibrio cholera, 63\% for Vibrio vulnificus and 79\% for Vibrio parahaemolyticus. Out of this study the most common Vibrio to cause severe wound infections was found to be Vibrio vulnificus. The brackish water of the Baltic seems to favor the occurrence of Vibrio vulnificus. Virulence genes specific to Vibrio vulnificus and Vibrio parahaemolyticus were present in about half of the samples, indicating a potential risk of catching infections for susceptible people exposed to the sea water.

A retrospective questionnaire with the intention to investigate the clinical course and epidemiology of Vibrio vulnificus infections in Japan was preformed surveying 1,693 hospitals around the country. Out of those, 1,045 did respond. Only 94 cases had been confirmed between 1999 and 2003. The $72.3 \%$ of the cases had septic infection with a mortality rate of $75 \%$. All occurred between the months of June to November. Seventy-seven patients had liver function impairments, and 53 had liver cirrhosis as underlying disease [6].

A study was conducted by Matsuoka et al of 12 cases including eight men and four women above the age of 50 with underlying diseases. Eight of these patients developed symptoms between June and September. During the initial medical examination, all patients exhibited fever, sharp pain with redness, swelling, and purple spots and blisters. Only one of these patients exhibited full-blown necrosis. In laboratory findings, all had systemic inflammatory response syndrome and sepsis, hepatic dysfunction, renal disorders and clotting abnormalities.

Treatment of infection by Vibrio vulnificus can be complicated because of its rapid onset. The sooner the treatment starts, the better. Studies have shown sensitivity to tetracycline, aminoglycosides, cephalosporins (third generation), chloramphenicol and newer fluroquinolones. However, two specific strains (CMCP6 and YJ016) that contain enzymatic-modification systems have antibiotic resistance. A study conducted by Craig in 2008 screened 151 coastal isolates with 141 local and 10 systemic cases. Sensitivities against 26 antimicrobial agents were measured, and up to $17.3 \%$ of isolates were resistant to eight or more antimicrobial agents. In general, mixed infections should be treated with ampicillin/sulbactam or cefoxitin. An alternative for sepsis cases would be a combination of clindamycin/ metronidazole plus gentamicin plus ampicillin that will cover a broad range of aerobes and anaerobes.

Early and aggressive surgical exploration is essential in patients with necrotizing fasciitis, myositis or gangrene to remove necrotic tissue and reduce the chances for compartment syndrome. It will also help by providing material for gram stain and cultures. Hyperbaric oxygen treatment might also be beneficial, but no prospective studies are available.

\section{Conclusion}

Necrotizing fasciitis due to Vibrio vulnificus is a sever disease with rapid progression and poor outcomes. It should be in mind in coastal regions and cases of marine contacts. Delay in diagnosis and timely treatment can have devastating outcomes.

\section{Conflict of Interest}

No conflict of interest.

\section{Funding}

No funding for this paper.

\section{References}

1. Zaidenstein R, Sadik C, Lerner L, Valinsky L, Kopelowitz J, Yishai R, Agmon V, et al. Clinical characteristics 
and molecular subtyping of Vibrio vulnificus illnesses, Israel. Emerg Infect Dis. 2008;14(12):1875-1882.

2. Fazeli MS, Keramati MR. Necrotizing fascitis: an epidemiologic study of 102 cases. Indian J Surg. 2007;69(4):136-139.

3. Baker-Austin C, McArthur JV, Lindell AH, Wright MS, Tuckfield RC, Gooch J, Warner L, et al. Multi-site analysis reveals widespread antibiotic resistance in the marine pathogen Vibrio vulnificus. Microb Ecol. 2009;57(1):151159.

4. Chuang YC, Yuan CY, Liu CY, Lan CK, Huang AH. Vi- brio vulnificus infection in Taiwan: report of 28 cases and review of clinical manifestations and treatment. Clin Infect Dis. 1992;15(2):271-276.

5. Collins B, Rehnstam-Holm A. Occurrence and potential pathogenesis of vibrio cholera, Vibrio parahaemolyticus and vibrio vulnificus on the south coast of Sweden. FEMS. 2011.

6. Inoue $\mathrm{Y}$, Ono $\mathrm{T}$, Matsui $\mathrm{T}$, Miyasaka J, Kinoshita $\mathrm{Y}$, Ihn H. Epidemiological survey of Vibrio vulnificus infection in Japan between 1999 and 2003. J Dermatol. 2008;35(3):129-139. 